\title{
Resposta quantitativa do florescimento da soja à temperatura e ao fotoperíodo(1)
}

\author{
Osmar Rodrigues ${ }^{(2)}$, Agostinho Dirceu Didonet(2), Júlio César Barreneche Lhamby ${ }^{(2)}$, \\ Paulo Fernando Bertagnolli(2) e Juliana Silva da Luz ${ }^{(3)}$
}

\begin{abstract}
Resumo - A previsão da data de floração da soja é importante para o manejo da cultura e para o uso em modelos de crescimento e de produção. O objetivo deste estudo foi o de quantificar o efeito do fotoperíodo e da temperatura na duração do período de florescimento, e avaliar a resposta de um modelo linear simples para predizer o período de floração de genótipos de soja de diferentes grupos de maturação, em diferentes épocas. Foi avaliada a habilidade da equação $\mathrm{D}=\mathrm{a}^{\prime}+\mathrm{b}^{\prime} \mathrm{T}+\mathrm{c}^{\prime} \mathrm{F}$, onde a', b' e c' são parâmetros empíricos, e T e F representam a média da temperatura e do fotoperíodo entre a emergência e a floração. Os parâmetros no modelo foram avaliados mediante análise de regressão múltipla, combinando os dados de dois anos agrícolas, em Passo Fundo, RS, em relação a cada genótipo. O período entre a emergência e o florescimento foi afetado pela temperatura e pelo fotoperíodo. As cultivares e a linhagem apresentaram diferentes sensibilidades a cada um desses fatores, resultando em coeficientes distintos. As datas de floração estimadas e observadas foram altamente significativas $\left(\mathrm{r}^{2}\right.$ superiores a 0,77$)$ em todos os genótipos, com uma variação de erro-padrão entre 2,4 e 4,8 dias.
\end{abstract}

Termos para indexação: Glycine max, floração, fatores ambientais, época de semeadura.

\section{Quantitative response of soybean flowering to temperature and photoperiod}

\begin{abstract}
Predicting the time of soybean flowering is a critical step for crop management practices, as well as for the development of crop models. The objective of this study was to quantify the effect of photoperiod and of temperature on the duration of the flowering period, and to evaluate the response of a simple linear model for predicting the flowering period of soybean genotypes of different maturation groups within different epochs. The ability of the equation $\mathrm{D}=\mathrm{a}^{\prime}+\mathrm{b}^{\prime} \mathrm{T}+\mathrm{c}^{\prime} \mathrm{F}$, where $\mathrm{a}^{\prime}$, b' and c' are empirical parameters, and $\mathrm{T}$ and $\mathrm{F}$ refer to the medium temperature and to the photoperiod between emergence and flowering, was evaluated. Evaluation of the coefficients were carried out through multiple regression analysis combining the data sets from two growing seasons for each genotype (1995/96 and 1996/97) in Passo Fundo, RS, Brazil. Multiple regression analysis showed that the period between emergence and flowering was influenced by temperature and photoperiod. The cultivars under study showed different susceptibilities to each factor, resulting in specific coefficients. The agreement between observed and predicted time of flowering was highly significant for all genotypes ( $\mathrm{r}^{2}$ higher than 0.77 ) with standard deviations ranging from 2.4 and 4.8 days.
\end{abstract}

Index terms: Glycine max, flowering, environmental factors, sowing date.

(1) Aceito para publicação em 2 de junho de 2000

(2)Embrapa-Centro Nacional de Pesquisa de Trigo, Caixa Postal 451, CEP 99001-970 Passo Fundo, RS. E-mail: osmar@cnpt.embrapa.br, didonet@cnpt.embrapa.br, julio@cnpt.embrapa.br, bertag@enpt.embrapa.br

(3)Universidade de Passo Fundo (UPF), Instituto de Ciências Biológicas, Bairro São José s/n, CEP 99001-970 Passo Fundo, RS. E-mail: icb@ufpel.tche.br

\section{Introdução}

Fotoperíodo e temperatura são importantes para o desenvolvimento da cultura da soja, por provocarem mudanças qualitativas ao longo do seu ciclo. As respostas a esses dois fatores não são lineares durante o ciclo de vida da cultura, pois existem 
subperíodos em que ela é incapaz de perceber esses sinais. Vários estudos têm caracterizado esses subperíodos, quanto à sensibilidade a temperatura $\mathrm{e}$ a fotoperíodo, principalmente entre a emergência e a floração (Major et al., 1975; Jones \& Laing, 1978; Hodges \& French, 1985; Wilkerson et al., 1989).

O subperíodo vegetativo pode ser definido como pré-indutivo ou juvenil. Nesse período, entre a emergência e a primeira folha verdadeira (estádio $V_{1}$ ) as plantas de soja são incapazes de perceber estímulo ao fotoperíodo. A partir daí, dependendo do genótipo, a planta adquire a capacidade de perceber os estímulos (maturação), que induzem as transformações de seus meristemas vegetativos em reprodutivos (fase indutiva). Com o início da diferenciação do primórdio floral até a floração (antese) (subperíodo pós-indutivo), ocorre o desenvolvimento dos primórdios florais (organogênese floral). A duração desses subperíodos é determinada pelo grau de sensibilidade termofotoperiódica do genótipo. Assim, em dias longos, a taxa de desenvolvimento dos órgãos reprodutivos é menor, e em baixas temperaturas ocorre uma diminuição no número de primórdios reprodutivos e na taxa de desenvolvimento. Dessa forma, a data de antese é uma avaliação fenológica importante, podendo ser relacionada com o tamanho da planta. Contudo, é a transformação das gemas vegetativas apicais em reprodutivas a causa da finalização da geração das estruturas vegetativas.

A sensibilidade fotoperiódica varia com 0 genótipo, e o grau de resposta ao estímulo fotoperiódico é o principal determinante da área de adaptação das diferentes cultivares. Nas cultivares de soja sensíveis, a resposta ao fotoperíodo é quantitativa, e não absoluta, o que significa que a floração ocorrerá de qualquer modo. No entanto, o tempo requerido para tal dependerá do comprimento do dia, sendo mais rápida a indução com dias curtos do que com dias longos. Desse modo, a indução floral provoca a transformação dos meristemas vegetativos (diferenciação de talos e folhas) em reprodutivos (primórdios florais), determinando o tamanho final das plantas (número de nós) e portanto seu potencial de rendimento. Cultivares de maturação tardia são geralmente mais sensíveis ao fotoperíodo do que cultivares precoces (Lawn \& Byth, 1973; Major et al., 1975 ).
Há muito tempo já se conhece que o intervalo de tempo, em número de dias entre a emergência e o florescimento, depende da influência da temperatura e do fotoperíodo, e que existe determinado limite de comprimento de dia suficientemente curto para induzir a floração e suficientemente longo para impedi-la. Este é caracterizado como fotoperíodo crítico (Steinberg \& Gardner, 1936). O comprimento do fotoperíodo crítico varia também entre cultivares de soja (Johnson et al., 1960).

Com relação à temperatura, Garner \& Allard (1930) concluíram que em ambientes com fotoperíodo constante ela influencia grandemente o tempo de florescimento. Segundo Pascale (1969), existe uma relação inversa entre a temperatura média e o número de dias necessários para a floração. Dessa forma, temperaturas mais baixas causam aumento no período para que ocorra o florescimento. Parker $\&$ Borthwick (1943) observaram que a indução floral foi ótima quando a temperatura nas folhas estava entre 21 e $27^{\circ} \mathrm{C}$ à noite, e que acima dos $27^{\circ} \mathrm{C}$ poucos primórdios florais foram formados.

$\mathrm{O}$ efeito do fotoperíodo e da temperatura no período de florescimento em soja tem sido estudado por meio de uma relação quantitativa entre essas variáveis. Major et al. (1975) usaram um modelo multiplicativo da temperatura e fotoperíodo para descrever o período de florescimento da soja. Hodges \& French (1985) usaram uma técnica estatística para obter uma equação capaz de prever a data de florescimento em soja. Por sua vez, Sinclair et al. (1991) utilizaram modelos linear e logístico, com base na temperatura e no fotoperíodo, para prever a data de florescimento de cultivares de soja de vários grupos de maturação. Ambos os modelos deram acurada previsão da data de florescimento das cultivares.

A previsão da data de floração, bem como de outros estádios de desenvolvimento em soja, é de suma importância para o manejo da cultura, como também para uso em modelo de crescimento e produção de soja. A correta previsão da duração entre a emergência e a floração determina ainda a produção de matéria seca, e, conseqüentemente, a produção de grãos (Shanmugasundaram \&Tsou, 1978; Wang et al., 1997). Além disso, pode fornecer indicações sobre como manejar a cultura para escapar de pe- 
ríodos de estresse característicos (falta de água, acamamento) em alguns ambientes, em determinadas regiões de cultivo de soja.

A previsão do comportamento fenológico em soja é dificultada pela falta de entendimento da influência dos fatores de ambiente no seu desenvolvimento vegetativo e reprodutivo. Contudo, a grande facilidade com que os parâmetros dos modelos de regressão podem ser estimados constitui uma atrativa opção para previsão da data de floração.

A utilização do conceito da taxa de desenvolvimento (inverso do tempo de duração) desenvolvido por Wit et al. (1970) foi um importante avanço na previsão do comportamento fenológico da cultura de soja. Hadley et al. (1984) usaram este conceito para definir a taxa de desenvolvimento como o inverso do tempo entre a emergência e o florescimento (1/f). Dessa forma, uma cultivar com longo período da emergência à floração (f: dias) teria uma pequena taxa de desenvolvimento (D: dias ${ }^{-1}$ ). Assim, pode-se analisar o comprimento do período por meio do $\mathrm{D}$ como função linear e aditiva da temperatura $(\mathrm{T})$ e do fotoperíodo (F) médios para o referido período, pela equação $1 / f=D=a^{\prime}+b^{\prime} T+c^{\prime} F$, onde: $a^{\prime}$, b' e c' são coeficientes empíricos. Os valores de $\mathrm{T}$ e F representam a média da temperatura e fotoperíodo entre a emergência e a floração (Hadley et al., 1984).

O objetivo deste trabalho foi quantificar o efeito do fotoperíodo e da temperatura na duração do período de florescimento, e avaliar a resposta de um modelo linear simples para predizer o período de floração de genótipos de soja, de diferentes grupos de maturação, semeados em épocas diferentes.

\section{Material e Métodos}

As avaliações fenológicas foram realizadas nos anos agrícolas 1995/96 e 1996/97, na área experimental da Embrapa-Centro Nacional de Pesquisa de Trigo (CNPT), em Passo Fundo, RS (latitude: $28^{\circ} 15^{\prime} \mathrm{S}$, longitude $52^{\circ} 24^{\prime} \mathrm{W}$ e $687 \mathrm{~m}$ de altitude). O solo onde foi conduzido este estudo é do tipo Latossolo Vermelho-Escuro distrófico (LE), com as seguintes características $(0-20 \mathrm{~cm})$ : no ano agrícola de 1995/96 $\left(\mathrm{pH}_{\mathrm{H}_{2} \mathrm{O}}=5,7\right.$; $\mathrm{pH}_{\mathrm{SMP}}=5,8 ; \quad \mathrm{Al}^{3+}=0,5 \mathrm{mmol}_{\mathrm{c}} \mathrm{dm}^{-3} ; \mathrm{Ca}^{2+}=$ $46,6 \mathrm{mmol}_{\mathrm{c}} \mathrm{dm}^{-3} ; \mathrm{Mg}^{2+}=30 \mathrm{mmol}_{\mathrm{c}} \mathrm{dm}^{-3} ; \mathrm{P}=15,5 \mathrm{mg} \mathrm{kg}^{-1}$; $\mathrm{K}=120 \mathrm{mg} \mathrm{kg}^{-1}$; e $\left.\mathrm{MO}=26 \mathrm{~g} \mathrm{~kg}^{-1}\right)$, e no ano agrícola de $1996 / 97,\left(\mathrm{pH}_{\mathrm{H}_{2} \mathrm{O}}=5,8 ; \mathrm{pH}_{\mathrm{SMP}}=6,2 ; \mathrm{Al}^{3+}=\right.$
$1,25 \mathrm{mmol}_{\mathrm{c}} \mathrm{dm}^{-3} ; \mathrm{Ca}^{2+}=41 \mathrm{mmol}_{\mathrm{c}} \mathrm{dm}^{-3} ; \mathrm{Mg}^{2+}=$ $23,5 \mathrm{mmol}_{\mathrm{c}} \mathrm{dm}^{-3} ; \mathrm{P}=22 \mathrm{mg} \mathrm{kg}^{-1} ; \mathrm{K}=123 \mathrm{mg} \mathrm{kg}^{-1}$; e $\mathrm{MO}=22 \mathrm{~g} \mathrm{~kg}^{-1}$ ). Foram usadas nove cultivares de soja (Tabela 1) nos dois anos de estudo, semeadas em cinco épocas. No ano 1995/96, as datas de semeadura foram: $17 / 9,11 / 10,16 / 11,12 / 12$ e 5/1, e em 1996/1997: 18/9, 17/10, 12/11, 17/12 e 17/1. As avaliações foram realizadas mediante três observações semanais, nos seguintes estádios de crescimento: 1) emergência, definida pelo aparecimento dos cotilédones acima da superfície do solo (Em); e 2) R1: início da floração, de acordo com a escala proposta por Fehr et al. (1971). As cultivares e a linhagem foram reunidas de acordo com o grupo de maturação, para uma melhor caracterização (Tabela 1).

O ensaio foi estabelecido sob sistema plantio direto, com densidade de 300.000 plantas ha $^{-1}$, em espaçamento de $0,5 \mathrm{~m}$ entre fileiras. Cada unidade experimental foi constituída de oito linhas de $5 \mathrm{~m}$ de comprimento, distribuídas em blocos ao acaso, com quatro repetições A adubação de $\mathrm{P}$ e $\mathrm{K}$ foi efetuada antes da semeadura, conforme recomendações técnicas para a cultura de soja (1995/1996), usando-se $300 \mathrm{~kg} \mathrm{ha}^{-1}$ de fertilizante, da formulação N-P-K 0-20-30. Foi realizada aplicação de inseticida e de herbicida para controle de pragas e plantas indesejadas. Durante o período de estudo, nos dois anos agrícolas, os experimentos foram irrigados quando necessário, para evitar defíciência hídrica nas plantas.

As informações sobre temperatura e fotoperíodo correspondentes aos subperíodos foram fornecidas pela Estação Meteorológica, a $100 \mathrm{~m}$ do local do experimento, junto à Embrapa-CNPT. A temperatura média diária foi obtida pela média entre a temperatura mínima e máxima

Tabela 1. Intervalo de tempo entre a emergência e a floração (R1) de cultivares e da linhagem de soja de diferentes grupos de maturação, nas safras agrícolas de 1995/96 e 1996/97, em Passo Fundo, RS.

\begin{tabular}{lccc}
\hline $\begin{array}{l}\text { Cultivares } \\
\text { e linhagem }\end{array}$ & Grupo de maturação $^{(1)}$ & $\begin{array}{c}\text { Tempo para o florescimento } \\
\text { (dias) }\end{array}$ \\
\cline { 3 - 4 } & & Mínimo & Máximo \\
\hline FT Cometa & V & 33 & 52 \\
Ocepar 6 & VI & 40 & 63 \\
Ocepar 3 & V-VI & 40 & 67 \\
BR 16 & VI-VII & 38 & 67 \\
Ocepar 14 & VI-VII & 40 & 69 \\
FT Abyara & VIII & 42 & 69 \\
Ocepar 8 & PJL & 43 & 80 \\
BR 83147 & PJL & 47 & 80 \\
Ocepar 9 & PJL & 54 & 88 \\
\hline
\end{tabular}

${ }^{(1)}$ Grau de sensibilidade ao fotoperíodo, em escala crescente (de 00, 0, I, II, III, até o grupo de maturação X) de requerimento em dias curtos, originária dos EUA. (2)Período juvenil longo (genótipos com sensibilidade fotoperiódica modificada, ou seja, floração atrasada nas condições de dias curtos). 
O fotoperíodo foi calculado para a latitude de $28^{\circ} 15^{\prime} \mathrm{S}$, incluindo crepúsculo civil, de acordo com List (1951).

A avaliação dos coeficientes no modelo linear foi realizada mediante análise de regressão linear múltipla, combinando os dados dos dois anos agrícolas (1995/96 e 1996/97), referentes a cada cultivar. A temperatura média (T) e o fotoperíodo médio (F) foram calculados entre as datas correspondentes à emergência e o início da floração (R1). As datas foram analisadas somente em termos da equação: $D=a^{\prime}+b^{\prime} T+c^{\prime} F$, onde: $a^{\prime}, b^{\prime}$ e c' são coeficientes empíricos, de acordo com Hadley et al. (1984), pois o limite mínimo da taxa de desenvolvimento $\left(0,01 \operatorname{dias}^{-1}\right)$ e fotoperíodo inferior a 12 horas, não foram obtidos no presente estudo, o que indica este modelo como adequado às condições de ambiente do experimento. O valor mínimo de $\mathrm{D}$ obtido neste estudo foi de $0,011$ ( $1 / \mathrm{D}<88$ dias $)$, e o fotoperíodo mínimo nos quais as plantas estavam expostas foi de 13,2 horas.

A validação foi realizada mediante utilização da data de floração das cultivares FT Cometa, Ocepar 14, BR 16 e FT Abyara, obtidas em vários experimentos nos anos agrícolas de 1992/93, 1993/94, 1994/95, 1995/96, 1996/97 e 1997/98, no Município de Passo Fundo.

\section{Resultados e Discussão}

Houve grande variação entre os genótipos testados, com relação à duração do período entre a emergência e o início do florescimento, em que o tempo mínimo obtido foi de 33 dias, e o máximo, de 88 dias (Tabela 1).

O modelo de regressão linear utilizado para descrever os dados obtidos nos dois anos de estudo re- sultou em valores de $\mathrm{r}^{2}$ altamente significativos em todas as cultivares e na linhagem, indicando, assim, que o modelo foi adequado para descrever os processos (Tabela 2). Além disso, a análise de regressão múltipla revelou que o subperíodo entre a emergência e o florescimento foi afetado pela temperatura e pelo fotoperíodo médios.

As cultivares e a linhagem evidenciaram diferenças quanto à sensibilidade a essas variáveis (Tabela 2). Os valores do $\mathrm{r}^{2}$ foram mais baixos na cultivar mais precoce (FT Cometa) e nas duas cultivares com períodos juvenil longo (Ocepar 8 e 9). O baixo $\mathrm{r}^{2} \mathrm{da}$ cultivar FT Cometa, em relação aos demais, evidenciou maior dificuldade do modelo em descrever os dados, o que pode ser decorrente do curto fotoperíodo a que foi exposta, na mesma data de semeadura, impedindo a expressão da sensibilidade ao fotoperíodo pelo menos em parte do tempo. Nas cultivares com período juvenil longo, pelo menos parte do seu período não apresentou resposta ao fotoperíodo, e esse pode ter sido o motivo do baixo valor de $\mathrm{r}^{2}$, quando comparadas às demais. Contudo, a linhagem BR 83147, caracterizada por período juvenil longo, também não se comportou como os demais genótipos, ao apresentar o maior valor de $r^{2}$ observado no estudo.

De forma geral, a previsão do modelo linear proposto por Hadley et al. (1984) apresentou desempenho adequado, com valor de $\mathrm{r}^{2}$ superior a 0,77 nas cultivares e na linhagem de soja em estudo. Os errospadrão para comparação do modelo linear na previ-

Tabela 2. Parâmetros do modelo linear $\left(\mathrm{D}=\mathrm{a}^{\prime}+\mathrm{b}^{\prime} \mathrm{T}+\mathrm{c}^{\prime} \mathrm{F}\right)^{(1)}$, com base nas médias de fotoperíodo $(\mathrm{F})$ e temperatura (T) obtidos a partir das datas de floração das cultivares e da linhagem de soja, nas safras agrícolas de 1995/96 e 1996/97.

\begin{tabular}{lccccc}
\hline Cultivares e & \multicolumn{5}{c}{ Parâmetros } \\
\cline { 2 - 5 } linhagem & $\mathrm{a}$ & $\mathrm{b}$ & $\mathrm{c}$ & $\mathrm{r}^{2}$ & EP \\
\hline FT Cometa & 0,08419 & $-0,01045$ & 0,003914 & $0,78^{* *}$ & 3,3 \\
Ocepar 6 & 0,09250 & $-0,00978$ & 0,002892 & $0,89^{* *}$ & 2,4 \\
Ocepar 3 & 0,07323 & $-0,00848$ & 0,002937 & $0,87^{* *}$ & 2,8 \\
BR 16 & 0,12149 & $-0,01286$ & 0,003500 & $0,83^{* *}$ & 3,6 \\
Ocepar 14 & 0,09764 & $-0,01047$ & 0,003051 & $0,85^{* *}$ & 3,3 \\
FT Abyara & 0,11298 & $-0,01151$ & 0,002958 & $0,86^{* *}$ & 3,3 \\
Ocepar 8 & 0,08538 & $-0,00992$ & 0,003197 & $0,77^{* *}$ & 4,8 \\
BR 83147 & 0,08518 & $-0,00915$ & 0,002617 & $0,90^{* *}$ & 3,4 \\
Ocepar 9 & 0,05327 & $-0,00579$ & 0,001909 & $0,78^{* *}$ & 4,2 \\
\hline
\end{tabular}

${ }^{(1)} \mathrm{D}$ : taxa de desenvolvimento (recíproca do número de dias entre emergência de floração); $\mathrm{a}^{\prime}=\mathrm{d}^{-1} ; \mathrm{b}^{\prime}=\mathrm{h}^{-1} ; \mathrm{c}^{\prime}={ }^{\circ} \mathrm{C}^{-1} ; \mathrm{EP}=$ erro-padrão. ${ }^{* *}$ Significativo a $1 \%$ de probabilidade. 
são do florescimento, em relação aos dados observados de floração, variaram entre 2,4 a 4,8 dias (Tabela 2). Resultados semelhantes na predição da floração de nove e de três cultivares de soja foram obtidos, respectivamente, por Constable \& Rose (1988) e Summerfield et al. (1989). Em experimento de campo, Sinclair et al. (1991) testaram modelos linear e logístico para prever o florescimento em 12 cultivares de soja. Apesar de ambos os modelos terem apresentado desempenho adequado, o modelo linear revelou maior variação entre as datas observadas e calculadas. Conforme esses autores, a grande simplicidade do modelo linear e a facilidade na obtenção dos coeficientes por regressão linear múltipla tornam atrativo o seu uso na previsão da data de floração em soja.

A comparação dos coeficientes referentes a fotoperíodo (b') entre genótipos evidenciou que cultivares de maturação mais tardia (FT Abyara) e média (BR 16) apresentaram valores mais baixos, o que poderia indicar maior efeito inibitório de fotoperíodo longo na taxa de desenvolvimento dessas cultivares (Tabela 2). Quanto ao subperíodo em estudo, o fotoperíodo crítico observado entre genótipos foi menor nos de maturação tardia (FT Abyara e BR 16), o que indica que a taxa de desenvolvimento nessas cultivares deixou de responder ao fotoperíodo a valores mais baixos que as demais (Tabela 2).

O maior coeficiente de temperatura (c') foi observado na cultivar mais precoce (FT Cometa), o que indica que sua taxa de desenvolvimento foi maior com o aumento da temperatura, em comparação às demais. De forma geral, nas cultivares precoces, o efeito da temperatura é mais importante que o do fotoperíodo.

Entre as cultivares e a linhagem, caracterizadas por um período juvenil longo, observou-se uma variação em seus comportamentos. A cultivar Ocepar 9 apresentou maior retardamento à floração, sob estímulo ao desenvolvimento, maior coeficiente quanto ao fotoperíodo, e o menor, quanto à temperatura, entre todos os genótipos estudados. Tal comportamento é indicativo de grupo de maturação tardia. Wilkerson et al. (1989) também encontraram aumento substancial no período até floração, em uma linhagem de soja com período juvenil longo, em comparação com outras cultivares, mesmo quando as condições de crescimento foram favoráveis para alta taxa de desenvolvimento (temperaturas de $26^{\circ} \mathrm{C}$ e fotoperíodo de nove horas). Por outro lado, a cultivar Ocepar 8 e a linhagem BR 83147, também caracterizadas por período juvenil longo, mostraram índices de fotoperíodo e de temperatura muito semelhantes aos das demais cultivares, e diferentes em relação aos da cultivar Ocepar 9. Sinclair et al. (1991) observaram, em uma linhagem com período juvenil longo (PI 159925), que os coeficientes (b'e c') dos modelos estudados eram indicativos de cultivares precoces. Esse comportamento adverso entre esse grupo de genótipos pode representar a diferença de sensibilidade deles ao fotoperíodo indutivo experimentado. Neste sentido, até mesmo a fase juvenil tem sido recentemente apontada como sensível ao fotoperíodo (Wang et al., 1998), e mostrou que a taxa de desenvolvimento depende do fotoperíodo experimentado. Dessa forma, e independentemente do grande esforço da pesquisa, existem ainda dúvidas sobre a fase juvenil em soja, o que pode dificultar o entendimento do comportamento, no campo, das cultivares assim chamadas juvenis.

A relação entre a emergência e a floração das cultivares e da linhagem de soja foi linear e altamente significativa (Figura 1).

O efeito da temperatura e do fotoperíodo na taxa de desenvolvimento da cultivar Ocepar 14 é mostrado na Figura 2. Observa-se o grande efeito inibi-



Figura 1. Modelo linear para prever o número de dias entre a emergência e a floração em soja.

Pesq. agropec. bras., Brasília, v. 36, n. 3, p. 431-437, mar. 2001 
tório de fotoperíodos longos e baixas temperaturas na taxa de desenvolvimento.

O fotoperíodo crítico varia de acordo com a temperatura, tornando-se maior com o aumento dela. Nos genótipos em estudo, o fotoperíodo crítico foi estimado a partir do modelo estudado, e aumentou numa taxa de 16 minutos ${ }^{\circ} \mathrm{C}^{-1}$ na cultivar Ocepar 14 . Da mesma forma, a temperatura basal variou com o fotoperíodo, numa taxa de $3,4^{\circ} \mathrm{C} \mathrm{h}^{-1}$ de aumento no fotoperíodo, na mesma cultivar.

Os coeficientes desenvolvidos referentes a cada cultivar, no modelo, foram validados com datas de floração de outros experimentos, usando dados de quatro cultivares de diferentes grupos de maturação (FT Cometa, Ocepar 14, BR 16 e FT Abyara), em dois locais diferentes, em cinco anos distintos (Figura 3). Mesmo assim, apesar da grande faixa de combinação entre fotoperíodo e temperatura abrangida neste estudo mediante a da variação das datas de semeadura e validação com dados obtidos em vários anos em diferentes estudos, exigem-se cuidados na extrapolação, para outros genótipos, dos coeficientes obtidos nesse modelo. Quanto aos mesmos genótipos, cuidados semelhantes devem ser tomados, especialmente na extrapolação para ambientes, em que ocorrem variações substanciais no regime de temperatura e fotoperíodo (grandes áreas geográficas).

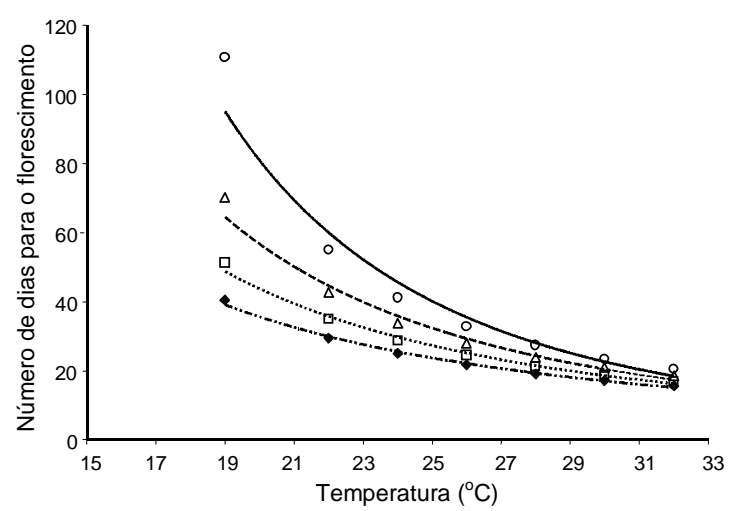

Figura 2. Número de dias para o florescimento da cultivar Ocepar 14 em razão da temperatura e do fotoperíodo $(\diamond 11$ horas, $\square 12$ horas, $\Delta 13$ horas, $\mathbf{O} 14$ horas ).



Figura 3. Validação do modelo linear para prever o número de dias entre a emergência e a floração (R1) em soja Os valores foram obtidos a partir de informações das cultivares, FT Cometa, Ocepar 14, BR 16 e FT Abyara, nos anos entre 1992 e 1997

\section{Conclusões}

1. O modelo de regressão linear múltipla é adequado para representar o período de duração (dias) entre a emergência e a floração.

2. A análise de regressão múltipla mostra que a duração do período entre a emergência e o florescimento é afetada pela temperatura e pelo fotoperíodo. Os genótipos apresentam diferentes tipos de sensibilidade a cada um desses fatores, resultando em coeficientes distintos em relação a cada cultivar e linhagem.

3. O fotoperíodo crítico e a temperatura basal variam com a temperatura e o fotoperíodo, respectivamente.

\section{Referências}

CONSTABLE, G. A.; ROSE, I. A. Variability of soybean phenology response to temperature, daylength and rate of change in daylength. Field Crops Research, Amsterdam, v. 18, p. 57-69, 1988

FEHR, W. R.; CALVINESS, C. E.; BURMOOD, D. T. PENNINGTON, J. S. Stage of development description for soybean, Glycine max (L.) Merrill. Crop Science, Madison, v. 11, p. 929-931, 1971 
GARNER, W. W.; ALLARD, H. A. Photoperiodic response of soybeans in relation to temperature and other environmental factors. Journal of Agricultural Research, Washington, v. 41, p. 719-735, 1930.

HADLEY, P.; ROBERTS, E. H.; SUMMERFIELD, R. J.; MINCHIN, F. R. Effects of temperature and photoperiod on flowering in soya bean [Glycine max (L.) Merril]: a quantitative model. Annals of Botany, London, v.53, p. 669-681, 1984

HODGES, T.; FRENCH, V. Soyphen: soybean growth stages modeled from temperature, daylength, and water availability. Agronomy Journal, Madison, v. 77, p. $500-505,1985$.

JONES, P. G.; LAING, D. R. Simulation of the phenology of soybeans. Agricultural Systems, Oxford, v. 3 , p. $295-311,1978$.

JOHNSON, H. W.; BORTHWICK, H. A.; LEFFEL, R. C. Effects of photoperiod and time of planting on rates of development of the soybean in various stages of the life cycle. Botanical Gazette, Chicago, v. 22, p. 77-95, 1960

LAWN, R. J.; BYTH, D. E. Response of soya beans to planting date in South-Eastern Queensland. I. Influence of photoperiod and temperature on phasic development patterns. Australian Journal of Agricultural Research, Collingwood, v. 24, p. 67-80, 1973.

LIST, R. J. Smithsonian meteorological tables. 6. ed Washington: Smithsonian Institution, 1951. $527 \mathrm{p}$ (Smithsonian Miscellaneous Collections, 114)

MAJOR, D. J.; JOHNSON, D. R.; TANNER, J. W.; ANDERSON, I. C. Effects of daylength and temperature on soybean development. Crop Science, Madison, v. 15, p. $174-179,1975$

PARKER, M. W.; BORTHWICK, H. A. Influence of temperature on photoperiodic reactions in leaf blades of Biloxi soybean. Botanical Gazette, Chicago, v. 104, p. $612-619,1943$
PASCALE, A. J. Tipos agroclimáticos para el cultivo de la soya en la Argentina. Revista de la Facultad de Agronomía e Veterinaria, Buenos Aires, v. 17, p. 31-38, 1969.

SHANMUGASUNDARAM, S.; TSOU, S. C. S. Photoperiod and critical duration for flower induction in soybean. Crop Science, Madison, v. 18, p. 598-601, 1978.

SINCLAIR, T. R.; KITANI, S.; HINSON, K.; BRUNIARD, J.; HORIE, T. Soybean flowering date: linear and logistic models based on temperature and photoperiod. Crop Science, Madison, v. 31, p. 786-790, 1991.

STEINBERG, R. A.; GARDNER, W. W. Response of certain plants to length of day and temperature under controlled conditions. Journal of Agricultural Research Washington, v. 52, p. 943-960, 1936.

SUMMERFIELD, R. J.; ROBERTS, E. H.; LAWN, R. J. Measurement and prediction of flowering in soybeans in fluctuating field environments. In: WORLD SOYBEAN RESEARCH CONFERENCE, 4., 1989, Buenos Aires. Proceedings... Buenos Aires : Argentina Soybeans Association, 1989. p. 82-87.

WANG, Z.; REDDY, R. V.; ACOCK, M. C. Testing for early photoperiod insensitivity in soybean. Agronomy Journal, Madison, v. 90, p. 389-392, 1998.

WANG, Z.; REDDY, R. V.; QUEBEDEAUX, B. Growth and photosynthetic responses of soybean to short-term cold temperature. Environmental and Experimental Botany, W. Conshohocken, v. 37, p. 13-24, 1997.

WILKERSON, G. G.; JONES, J. W.; BOOTE, K. J.; BUOL, G. S. Photoperiodically sensitive interval in time to flower of soybean. Crop Science, Madison, v. 29 , p. $721-726,1989$.

WIT, C. T. de; BROUWER, R.; VRIES, F. W. T. P. der. The simulation of photosynthetic systems. In : SETLIK, I. (Ed.). Prediction and measurement of photosynthetic productivity. Wageningen : PUDOC, 1970. p. 47-70. 